\title{
Podemos falar de segurança do paciente durante uma pandemia? Uma experiência portuguesa
}

\author{
Can we talk about patient safety during a pandemia? \\ A portuguese experience
}

¿Podemos hablar sobre seguridad del paciente durante una pandemia?

Una experiencia portuguesa

\author{
Catarina Isabel Gomes Mendes Ferreira ${ }^{1}$ \\ Ana Cristina de Almeida Marinho Diniz ${ }^{2}$ \\ Idalina Maria Santos Vieira Lisboa Bordalo ${ }^{3}$ \\ Maria João Lage de Sousa Leitão ${ }^{4}$ \\ Susana Maria Sardinha Vieira Ramos ${ }^{5}$
}

\begin{abstract}
Resumo
Objetivo: enumerar medidas governativas e de saúde pública implementadas em Portugal decorrentes da pandemia pelo vírus SARS-CoV-2 e descrever a atuação do Gabinete de Segurança do Paciente do Centro Hospitalar Universitário de Lisboa Central (CHULC), referência nacional na resposta à situação de emergência de saúde pública. Métodos: utilizou-se como método a análise normativa, documental, além de relato de caso por pesquisa-ação. Resultados: em Portugal, documentaram-se os primeiros casos de infeção por SARS-CoV-2 a 2 de março de 2020. Os processos assistenciais são geridos pelo Ministério da Saúde e Direção Geral da Saúde, abrangendo o Sistema Nacional de Saúde (universal) e o sector privado. O Gabinete de Segurança do Doente do CHULC, participou na redefinição dos processos, criação de vias alternativas de informação entre as várias estruturas, implementação de inovações no uso de tecnologias e vigilância clínica, gestão dos equipamentos de proteção, motivação e suporte emocional dos profissionais e na consolidação das principais metas de segurança do doente (ex. identificação do doente, medicação e cirurgia segura). A aprendizagem feita com os erros contribui para a melhoria contínua dos processos. Conclusão: em Portugal e no mundo a pandemia por COVID-19 não terminou. Compreendemos que é tempo de refletir e voltar aos princípios básicos da segurança, como a higiene das mãos, a etiqueta respiratória e o controlo ambiental. $\mathrm{Na}$
\end{abstract}

\footnotetext{
${ }^{1}$ Mestre em Enfermagem, Universidade Católica Portuguesa, Lisboa, Portugal; enfermeira especialista e gestora do risco em saúde, Gabinete de Segurança do Doente, Centro Hospitalar Universitário de Lisboa Central, Lisboa, Portugal. https://orcid.org/0000-0003-3782-4057. E-mail: catarina.mendes@chlc.min-saude.pt

2 Mestre em Enfermagem, Universidade Católica Portuguesa, Lisboa, Portugal; enfermeira especialista e gestora do risco em saúde, Gabinete de Segurança do Doente, Centro Hospitalar Universitário de Lisboa Central, Lisboa, Portugal. https://orcid.org/0000-0001-9762-9785. E-mail: ana.diniz@chlc.min-saude.pt

${ }^{3}$ Mestre em Gestão dos Serviços de Saúde, Instituto para o Desenvolvimento Empresarial e Gestão/Instituto Superior de Ciências do Trabalho e Empresa, Lisboa, Portugal; enfermeira especialista e gestora do risco em saúde, Gabinete de Segurança do Doente, Centro Hospitalar Universitário de Lisboa Central, Lisboa, Portugal. https://orcid.org/0000-00031404-7443. E-mail: idalina.bordalo@chlc.min-saude.pt

${ }^{4}$ MSc Quality and Patient Safety in Healthcare, Imperial College, Londres, Reino Unido; assistente hospitalar e coordenadora, Gabinete de Segurança do Doente, Centro Hospitalar Universitário de Lisboa Central, Lisboa, Portugal. https://orcid.org/0000-0002-3968-4804. E-mail: jjlage@chlc.min-saude.pt

${ }_{5}^{5}$ Mestre em Infeções Relacionadas com os Cuidados de Saúde, Universidade Católica Portuguesa, Lisboa, Portugal; enfermeira gestora e coordenadora, Gabinete de Segurança do Doente, Centro Hospitalar Universitário de Lisboa Central, Lisboa, Portugal. https://orcid.org/0000-0003-4043-7955. E-mail: susana.ramos@chlc.min-saude.pt
} 


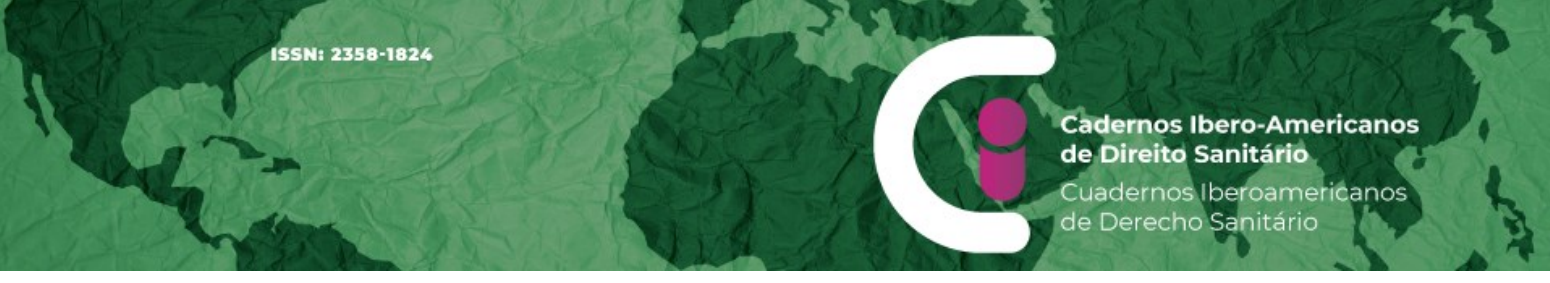

gestão do risco e segurança do paciente em situação de crise, é necessário, mais do que nunca, inovar e aprender com os erros.

\title{
Palavras-chave
}

Pandemias. Portugal. Segurança do paciente.

\begin{abstract}
Objective: to enumerate governmental and public health measures implemented in Portugal resulting from the SARS-CoV-2 virus pandemic and describe the work of the Patient Safety Office of the Centro Hospitalar Universitário de Lisboa Central (CHULC), a national reference in the response to the situation of public health emergency. Methods: normative, documentary analysis was used as a method, in addition to case reports by action research. Results: in Portugal, the first cases of SARS-CoV-2 infection were documented on March 2, 2020. Assistance processes are managed by the Ministry of Health and the General Directorate of Health, covering the National Health System (universal) and the private sector. The CHULC Patient Safety Office, participated in the redefinition of processes, creation of alternative information routes between the various structures, implementation of innovations in the use of technologies and clinical surveillance, management of protective equipment, motivation and emotional support from professionals and consolidating the main patient safety goals (eg patient identification, medication and safe surgery). Learning from mistakes contributes to the continuous improvement of processes. Conclusion: In Portugal and in the world, the COVID-19 pandemic has not ended. We understand that it is time to reflect and return to the basic principles of safety, such as hand hygiene, respiratory etiquette and environmental control. In the management of risk and safety of patients in crisis situations, it is necessary, more than ever, to innovate and learn from mistakes.
\end{abstract}

\section{Keywords}

Pandemics. Portugal. Patient safety.

\section{Resumen}

Objetivo: enumerar las medidas gubernamentales y de salud pública implementadas en Portugal como resultado de la pandemia del virus SARS-CoV-2 y describir el trabajo de la Oficina de Seguridad del Paciente del Centro Hospitalar Universitário de Lisboa Central (CHULC), una referencia nacional en la respuesta a la situación de emergencia de salud pública. Métodos: se utilizó como método el análisis documental, normativo, además de los reportes de casos por investigación-acción. Resultados: en Portugal se documentaron los primeros casos de infección por SARS-CoV-2 el 2 de marzo de 2020. Los procesos de atención son gestionados por el Ministerio de Salud y la Dirección General de Salud, abarcando el Sistema Nacional de Salud (universal) y el privado. sector. La Oficina de Seguridad del Paciente del CHULC, participó en la redefinición de procesos, creación de rutas alternativas de información entre las distintas estructuras, implementación de innovaciones en el uso de tecnologías y vigilancia clínica, manejo de equipos de protección, motivación y apoyo emocional de los profesionales y consolidando las principales objetivos de seguridad del paciente (por ejemplo, identificación del paciente, medicación y cirugía segura). Aprender de los errores contribuye a la mejora continua de los procesos. Conclusión: En Portugal y en el mundo, la pandemia de COVID-19 no ha terminado. Entendemos que es hora de reflexionar y volver a los principios básicos de seguridad, como la higiene de manos, la etiqueta respiratoria y el control ambiental. En la gestión del riesgo y la seguridad de los pacientes en situaciones de crisis, es necesario, más que nunca, innovar y aprender de los errores. 


\section{Palabras clave}

Pandemias. Portugal. Seguridad del paciente.

\section{Introdução}

Em Portugal, os primeiros casos de SARS-CoV-2 surgiram no início de março de 2020 (1). Desde há várias semanas que o País assistia às notícias do que se passava no resto do Mundo. Seria apenas uma questão de tempo, até que a infeção por SARS-CoV-2 chegasse a território português.

A 2 de Março, o Ministério da Saúde (MS) e a Direção-Geral da Saúde (DGS), numa conferência de imprensa conjunta, confirmavam o inevitável: os primeiros dois casos com diagnóstico de COVID-19 no País. Tratavam-se de dois cidadãos portugueses com viagens recentes ao estrangeiro, um ao Norte de Itália e outro à região de Valência, em Espanha.

Foi assim definido um conjunto de medidas legislativas para fazer face à pandemia, sendo as primeiras trinta medidas divulgadas a 13 março (2). De entre elas, salienta-se o encerramento de várias instituições públicas e privadas, e o impacto daí decorrente, nomeadamente no que diz respeito ao encerramento de escolas e creches. Simultaneamente foram também reorganizados serviços públicos e privados. Incentivou-se a implementação do teletrabalho em todas as situações em que tal fosse possível.

Empreenderam-se esforços para dar resposta ao conjunto das 10 medidas transversais aprovadas pela União Europeia para lutar contra a pandemia (3): limitar a propagação do vírus; fornecer equipamento médico; promover a investigação; garantir a recuperação da União Europeia; repatriar cidadãos europeus; solidariedade europeia; apoiar a economia; proteger os empregos; salvaguardar a internet e proteger o meio ambiente e as linhas aéreas. Outras medidas foram acordadas com o Governo espanhol, nomeadamente, o controlo das fronteiras terrestres (4). Igualmente oportuna foi a regularização de estrangeiros com pedidos pendentes, garantindo-lhes os mesmos direitos que aos cidadãos nacionais, em particular no acesso aos cuidados de saúde (5).

O direito universal a cuidados de saúde globais, elencado no artigo $64^{\circ}$ da Constituição da República Portuguesa (6), está concretizado na Lei 56/79 (7) que cria o Serviço Nacional de Saúde (SNS) português. A necessidade de garantir a capacidade de resposta do SNS tem sido o pilar central nas tomadas de decisão governativas. A prioridade tem sido, desde o início, aplanar a curva epidemiológica, de modo a assegurar cuidados de saúde, apesar do crescente número de casos confirmados. 
Apesar da emergência na resposta, não poderão ser negligenciados os constructos do Plano Nacional para a Segurança dos Doentes 2015-2020 (8). Este plano visa, através de ações transversais, como a cultura de segurança, a partilha do conhecimento e da informação e de ações dirigidas a problemas específicos, a prestação segura de cuidados de saúde em todos os níveis, num processo de melhoria contínua da qualidade do SNS. É da responsabilidade de cada organização de saúde a concretização e adaptação destes pressupostos à sua realidade específica.

A contribuição do Centro Hospitalar Universitário Lisboa Central (CHULC) na resposta à pandemia é ilustrativa da realidade portuguesa. Esta entidade reorganizou-se, reafectando antecipadamente áreas cínicas para aumentar a disponibilidade de cuidados a doentes com COVID-19. A opção pelo internamento domiciliário, em todas as situações clinicamente possíveis, afigurou-se uma estratégia importante na evicção da sobrelotação das suas unidades de internamento hospitalar. A necessidade de driblar recursos limitados e até indisponibilidade de Equipamentos de Proteção Individual (EPI), evidenciou a importância da existência de um sistema de segurança do paciente, que neste centro tem cerca de duas décadas de implementação. Este sistema inclui a identificação e avaliação do risco, bem como a notificação e análise de incidentes (9), suportados por um programa estruturado de formação em segurança do paciente (10). Estas estratégias têm permitido identificar diversos problemas e falhas latentes, a partir das quais se têm elaborado documentos e construído projetos de melhoria, ferramentas essenciais no controlo das falhas identificadas (11). Para além das questões mais óbvias relacionadas com o controlo de infeção, emergem entre outras, as dificuldades na comunicação (por exemplo pela utilização de EPI) e na identificação do paciente, bem como na preocupação com a segurança e bem-estar dos profissionais (12).

Num contexto em que a necessidade de adaptação às mudanças tem sido constante, o relato de casos e partilha de experiências tem contribuído para o desenvolvimento do conhecimento e de boas práticas. Deste modo, pretende-se com o presente artigo enumerar medidas governativas e de saúde pública implementadas em Portugal decorrentes da pandemia pelo vírus SARS-CoV-2, bem como descrever a atuação do Gabinete de Segurança do Paciente de um centro hospitalar, referência nacional na resposta à situação de emergência de saúde pública. A pandemia tirou-nos da zona de conforto, mas o objetivo comum de garantir a segurança de todos, levou a que novos talentos se revelassem. Foram criadas sinergias e cooperação entre áreas habitualmente mais afastadas. Estas mudanças 
são importantes na gestão da atual situação e podem aumentar a resiliência para eventos futuros (13).

\section{Métodos}

Para elaboração do presente artigo, foi realizado um levantamento bibliográfico de artigos obtidos em diferentes bases de dados de acesso gratuito, publicados em português e inglês. Utilizaram-se ainda, dados constantes em documentos oficiais disponibilizados nos sites do Diário da República, Ministério da Saúde e Direção Geral da Saúde (Portugal).

Complementarmente, optou-se pelo relato de caso por pesquisa-ação, dado o elevado envolvimento dos autores como participantes da pesquisa. Optou-se por esta metodologia uma vez que a pesquisa-ação é um processo emergente de investigação que integra teoria e ação para acoplar o conhecimento científico ao conhecimento organizacional existente e para abordar problemas organizacionais reais junto com as pessoas do sistema sob investigação (14).

\section{Relato da experiência de um centro hospitalar em Lisboa}

O CHULC integra o SNS tendo sido definido como um dos centros de referência de resposta a esta pandemia. Constituído por seis unidades hospitalares, emprega cerca de 8000 profissionais. Esta instituição disponibiliza cuidados de saúde diferenciados em várias especialidades, nomeadamente na área do coração, vasos e tórax; cirurgia e outras especialidades cirúrgicas; urgência e cuidados intensivos; neurociências; hemato-oncologia; ginecologia e obstetrícia; pediatria; músculo-esquelética; medicina interna.

Esta instituição de saúde tem mais de duas décadas de trabalho desenvolvido na área da Qualidade e Segurança do Paciente, sendo nacional e internacionalmente certificada e acreditada. Nos termos do Decreto Regulamentar $n^{\circ}$ 66/2007, de 29 de maio (15), o CHULC definiu um Plano de Catástrofe (16), onde a resposta a situações de pandemia está contemplada, desde 2008. Ao longo dos anos, tem feito atualizações periódicas e dinamizado várias estratégias, como por exemplo, simulacros para situações como o ebola e a gripe $\mathrm{H} 1 \mathrm{~N} 1$.

De acordo com as orientações publicadas pela DGS no Plano Nacional de Preparação e Resposta à Doença por Novo Coronavírus (COVID-19) (17), do European Center for Disease Prevention and Control (18) e da Organização Mundial de Saúde (19), no início de março de 2020, o Conselho de Administração (CA) avançou com medidas para dar resposta 
ao embate da primeira onda pandémica. Assim, vários elementos estratégicos da instituição foram convocados para integrar o Gabinete de Coordenação para o combate à COVID-19, o qual passou a reunir três vezes por semana para avaliar, discutir e procurar responder às necessidades identificadas. Devido ao aumento do número de casos, a estratégia de atuação passou a ser monitorizada diariamente pelos membros que integram este gabinete.

As duas Unidades de Infeciologia (pediátrica e de adultos) foram os locais de internamento dos primeiros doentes diagnosticados com COVID-19 na região de Lisboa. Rapidamente o número de doentes internados foi aumentando, validando a noção de que seria necessário reorganizar todo o centro hospitalar, desde os três serviços de urgência (pediátrica, ginecológica/obstétrica e de adultos), passando pelas unidades de internamento, blocos operatórios, cuidados intensivos e até a área ambulatória.

O principal enfoque foi sempre a segurança de todos, doentes $(20,21,22)$ e profissionais $(23,24,25,26)$. A necessidade de garantir a assistência e acompanhamento de todas as outras situações de doença que continuaram naturalmente a surgir, foi também uma das principais preocupações, e um grande desafio. A reorganização da atividade assistencial presencial, potenciou a utilização da telessaúde. Esta estratégia encontra-se agora por força da pandemia, na primeira linha das mudanças a investir nos cuidados de saúde. Outra área que ganhou relevância na realidade portuguesa foi a hospitalização domiciliária (27). Também esta nova realidade de saúde pública, veio evidenciar a sua pertinência, bem como a necessidade de um maior desenvolvimento desta forma de cuidar e de envolver os pacientes e seus cuidadores informais $(28,29)$.

A redefinição de processos assistenciais com a separação de circuitos de doentes suspeitos ou confirmados de COVID-19, dos restantes foi outra das preocupações, requerendo uma triagem robusta no momento da admissão dos doentes na instituição (30, $31,32)$.

\section{Inovar no cuidar}

Todas as Unidades se reinventaram. Criaram ou reorganizaram circuitos de doentes e profissionais; algumas fizeram obras e em tempo recorde, construíram soluções para os novos problemas, outras mudaram inclusive o seu âmbito assistencial. Reinventaram formas de comunicação e até de vigilância (33): colocando espelhos nas paredes para monitorizar os doentes reduzindo assim entradas nos quartos; dispondo monitores e dispositivos eletrónicos de perfusão nas antecâmaras ou corredores das enfermarias. Serviram refeições 
nos quartos aos pais das crianças internadas; sorrindo às crianças e adultos conscientes antes de colocar os equipamentos de proteção individual (EPI), através dos vidros das antecâmaras; ou ainda colocando uma fotografia sua e/ou o seu nome e categoria profissional nos EPI. Deram informações a familiares e proporcionando visitas virtuais com recurso a equipamentos doados, adquiridos pela instituição ou até dos próprios profissionais. Trouxeram doentes até às janelas das unidades para verem os entes queridos ainda que através dos vidros. $(34,35,36,37)$.

O acompanhamento do Conselho de Administração e órgãos de gestão e apoio técnico juntamente com as direções das várias áreas assistenciais foi fulcral na atualização dos planos de contingência de modo a garantir sua implementação. Outro aspeto determinante foi a gestão dos EPI e de outros dispositivos/equipamentos médicos, sendo necessário reforçar as unidades com maior afluência de doentes infetados. $O$ apelo à utilização adequada e racional dos EPI, evitando desperdícios, deu origem à frase: $E$ imprescindível o uso correto de equipamento proteção individual, proteja-se a si e aos outros, presente em todas as mensagens semanais da Presidente do Conselho de Administração.

\section{O poder da informação}

Desde cedo que o acesso à informação e orientações claras se tornou num dos maiores desafios. Todos os dias, e por vezes mais do que uma vez por dia, eram divulgadas orientações nacionais e institucionais, e nem todos os profissionais que estavam a cuidar de doentes tinham disponibilidade para acompanhar este ritmo vertiginoso. E é aqui que uma área de apoio e consultoria como o Gabinete de Segurança do Doente (GSD) pode dar o seu contributo. Mesmo à distância, por questões de segurança, a proximidade é possível, porque tal como escreveu um dos elementos da equipa do GSD, Ana Marinho: "A distância é relativa. E em tempos de pandemia a distância não existe! Vivemos e sentimos diferente, mas não de forma distante" (38).

A equipa do GSD começou por tentar saber o que mais precisavam os profissionais que estavam na linha da frente. E aqui, fez toda a diferença a rede e a estrutura criada desde há anos que integra elementos de cada unidade, designados como interlocutores do GSD. Informação - responderam. Informação é poder. Poder para estarem melhor preparados, para melhor decidirem e cuidarem.

A formação dos profissionais foi imperiosa até na gestão e controlo das suas emoções. O saber trouxe maior confiança, logo maior segurança. E foi por aí o caminho. 
Lendo, traduzindo e organizando informação recolhida sistematicamente em diversas fontes de referência nacionais e internacionais. Criando documentos concisos, mas com toda a informação essencial. (39)

Atendendo o telefone a qualquer hora, em qualquer dia da semana, a qualquer pessoa de qualquer grupo profissional. Articulando com as várias estruturas institucionais cujo papel de apoio técnico é também fundamental, e que integram a Comissão de Qualidade e Segurança do CHULC: Gabinete de Segurança do Doente; Gabinete de Gestão de Programas da Qualidade; Saúde Ocupacional; Grupo de Coordenação Local do Programa de Prevenção e Controlo de Infeção e Resistências Antibióticas; Gabinete de Segurança; Gabinete de Auditoria; Gabinete do Cidadão e Comissão de Catástrofe.

As situações de crise propiciam condições favorecedoras de falhas nos sistemas de trabalho, passíveis de originar erros que podem comprometer a segurança do paciente, nomeadamente a fadiga e burnout; falta de confiança; falta de tempo para prestar cuidados adequados e autocuidado; perceção de insegurança $(40,41)$.

Cientes desta realidade, procurou-se prevenir e controlar a transmissão da infeção por SARS-COV-2 em contexto hospitalar, mas não descurando outras áreas basilares neste âmbito. Assim, o apelo contínuo à implementação e até reforço de práticas seguras em todas fases do percurso do paciente foi uma das prioridades, nomeadamente na identificação inequívoca do paciente de modo a garantir o cuidado adequado à pessoa certa; segurança no circuito do medicamento e no circuito transfusional; segurança cirúrgica e na realização de exames complementares de diagnóstico e tratamento; na transição de cuidados e na comunicação entre profissionais; entre profissionais e pacientes/familiares. $(42,43,44,45,46)$.

Foram elaborados materiais informativos em formato multimédia e em cartazes com recomendações ilustrativas no âmbito da COVID-19 e da segurança do paciente de modo a facilitar a disseminação da informação e de boas práticas junto dos profissionais (47).

O Gabinete de Comunicação e Imagem foi fundamental no apoio, análise dos materiais em termos comunicacionais, na atualização e na organização do microsite criado para publicação de todo o material informativo relativo a esta temática. 


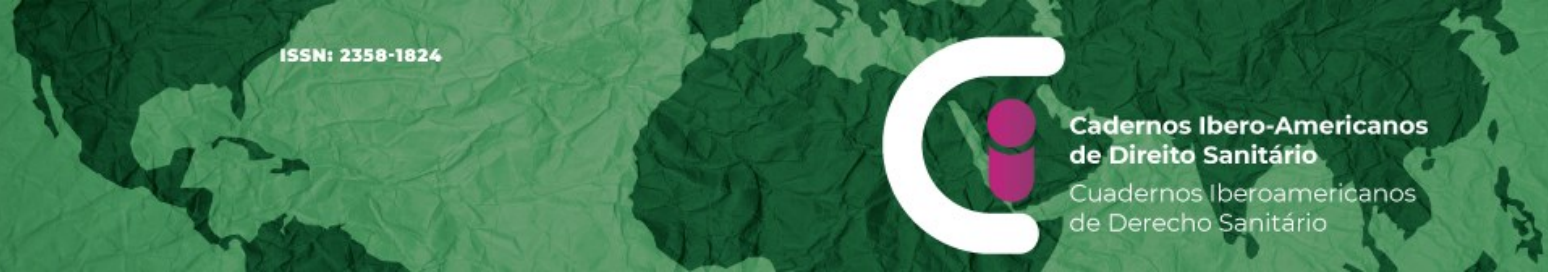

Figura 1. Cartaz informativo relativo a práticas seguras na identificação do doente no contexto da pandemia por COVID-19.

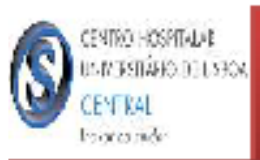

\section{IDENTIFICAÇÃO SEGURA DO DOENTE COVID 19}

A identificação inequívoca é fundamental, em qualquer circunstância, para a segurança do doente. Em situação de crise, as práticas seguras de identificação devem ser mantidas e até reforçadas para evitar erros.

Todos somos responsáveis por garantir os cuidados ao doente certo. A sistematização de medidas promotoras da segurança do doente faz a diferença e salva vidas!

\begin{tabular}{|l|l|}
\hline \multicolumn{2}{|c|}{ IDENTIFICAÇÃO POSITIVA } \\
\hline Pergunte ao Doente: & $\begin{array}{l}\text { Nome COMPLETO } \\
\text { DATA de nascimento }\end{array}$ \\
\hline
\end{tabular}

Confirme SEMPRE os dados na pulseira de identificação e o N.ํ do PROCESSO

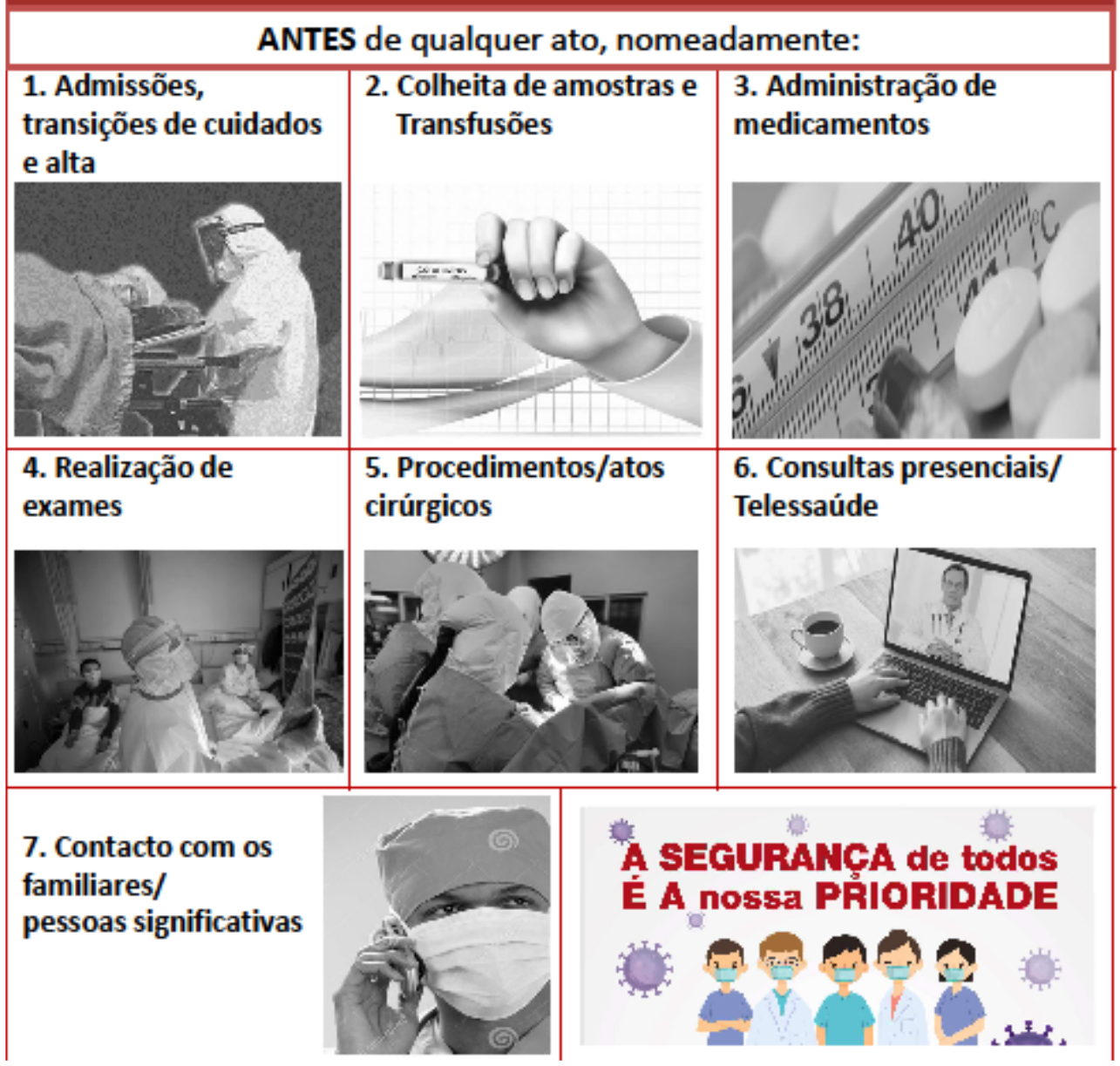

Fonte: Centro Hospitalar Universitário Lisboa Central (48). 
Tem sido assim possível dar resposta às solicitações do Conselho de Administração e das unidades, dos profissionais e dos pacientes, com o objetivo principal de criar confiança e promover a segurança de todos. Saber é poder, poder para estarem melhor preparados, para melhor decidirem e para melhor cuidarem (50).

Nesta nova caminhada da segurança do paciente em tempos adversos, recrearamse dinâmicas. Uma delas foi o lançamento de uma Linha de Atendimento, Informação e Orientação Técnica COVID-19 (LAIOT) (51). Esta ferramenta surgiu de uma parceria entre o Gabinete de Segurança do Doente e o Gabinete de Gestão de Projetos de Qualidade. Com a criação desta linha pretendeu-se facilitar o acesso rápido a informação relevante e atual permitindo aos profissionais resolver problemas em tempo útil; garantir o bem-estar das equipas e promover cuidados seguros, encontrando soluções criativas para além das necessidades imediatas. Tem como suporte uma base de dados, desenvolvida para o efeito, com listas de contactos, resumos de orientações internacionais, nacionais, institucionais e artigos científicos decorrentes de uma intensa pesquisa diária. O atendimento é realizado diariamente através de contacto telefónico ou através de e-mail. A equipa é constituída por enfermeiros e médicos e já atendeu mais de 1000 pedidos de apoio, sendo os motivos de contacto mais frequentes os relacionados com a saúde/vigilância do profissional, circuito do paciente e utilização de EPI.

\section{Bem estar físico, mental e social}

Nos contextos de emergência como a pandemia COVID-19, é normal o surgimento de sentimentos de ansiedade, medo, angústia e frustração $(52,53)$. Por esse motivo, o suporte emocional mereceu uma maior atenção no sentido de disponibilizar apoio aos profissionais, pacientes e famílias.

A saúde ocupacional, no âmbito das suas competências, mantém o acompanhamento dos profissionais, e em particular dos suspeitos ou infetados com COVID-19. Foi ainda delineada uma estratégia fundamental que consistiu na criação de uma linha de apoio emocional para os profissionais. Esta linha surge do trabalho conjunto da saúde ocupacional, das unidades de psicologia clínica e da psiquiatria e foi criada com o propósito de aumentar a resiliência dos profissionais e, simultaneamente dar-lhes apoio emocional (54), e em particular aos que contraíram infeção por SARS-CoV-2. Esta intervenção foi complementada com a publicação de vídeos motivacionais. 
A preocupação com a saúde mental e física dos profissionais concretizou-se ainda na dinamização de outra estratégia, desta vez pelas mãos de fisioterapeutas da instituição, que se disponibilizaram a realizar três sessões virtuais por semana, com atividades que vão desde o pilates clínico, ioga, fisiodance, treino funcional, entre outras. Esta atividade designada Pausa Ativa dirige-se a quem esteja a cumprir isolamento, ou se encontre em teletrabalho, tendo progressivamente sido alargada a todos os que mostraram interesse em participar.

Não é possível omitir a importância das relações interpessoais neste contexto marcado por medos, incertezas e inseguranças (40). Muitos profissionais destacam apesar das dificuldades, inúmeros ganhos, referindo que se estreitaram laços de companheirismo, se combateu o medo, a dúvida, com resiliência, coragem e espírito de equipa. Muitos sentiram que finalmente se concretizou o conceito de interdisciplinaridade (55). Também na relação com os pacientes, muitos referem que embora os contactos sejam agora menos frequentes, aproveitam melhor esse tempo, até para compensar a ausência do toque e a ausência dos seus entes queridos, medidas impostas para segurança de todos.

A restrição de visitas aos pacientes terá sido uma das mais difíceis decisões tomadas, ainda que se tenham considerado como situações de exceção, os pacientes pediátricos, parturientes, situações de pacientes com deficiência ou situação de dependência, com doença incurável em estado avançado e em estado final de vida. A estratégia imediata para ultrapassar o constrangimento das limitações de visitas, foi a disponibilização no site do CHULC, de uma lista de contactos telefónicos diretos para cada unidade. Os familiares/pessoas de referência, indicados pelo paciente, podem desta forma solicitar informações. Foi também estabelecido pelo CA que no processo clínico do paciente fica registado o contacto do familiar que fará este contacto telefónico.

Igualmente atenta a esta situação, a Unidade de Psicologia Clínica reorganizou a sua atividade e incluiu no seu plano de trabalho diário, o contacto telefónico para acompanhamento dos pacientes com diagnóstico de COVID-19 que estão em domicílio, para além do acompanhamento dos pacientes internados e seus familiares.

Apesar de se reconhecer a restrição de visitas como uma das medidas de combate à propagação desta doença, esta medida teve um grande impacto para todos $(56,57,58)$, contribuindo para o consequente afastamento entre doentes e familiares. Nesse sentido, foi criada no CHULC a Equipa Móvel Mais Próximos de Ti, constituída por profissionais deste centro hospitalar e, que de forma voluntária, conciliam a sua atividade atual com a desta 
equipa. Este projeto tem como principais objetivos apoiar e promover a comunicação e proximidade entre o paciente e os seus familiares, através de meios eletrónicos (59).

Analisando as estratégias desenvolvidas, é possível estabelecer uma correlação com as cinco etapas sugeridas por Staines et al (60), através das quais as competências das equipas responsáveis pela Qualidade e Segurança do Paciente, podem contribuir no processo de gestão da crise associada à pandemia:

- fortalecer o sistema através da avaliação da capacidade de resposta, recolhendo evidências, organizando informação e formação, promovendo a segurança das equipes, reforçando o apoio aos colegas;

- envolver os cidadãos, os pacientes e as suas famílias na procura conjunta de soluções;

- redefinir circuitos de atendimento, por meio de ações como a separação de fluxos, reuniões de trabalho em equipe e desenvolvimento de suportes à decisão clínica;

- reduzir potenciais danos através do gerenciamento proativo do risco para pacientes com COVID-19 e não-COVID-19; e

- impulsionar e expandir o sistema de aprendizagem, para identificar oportunidades de melhoria e agir a partir delas.

Para manter a qualidade e segurança da prestação de cuidados de saúde, perante tamanho desafio, é necessário priorizar o bem-estar e resiliência dos profissionais de saúde, para que estes sejam capazes de sentir que este pode ser uma oportunidade de aprendizagem e desenvolvimento, não só profissional, mas sobretudo pessoal.

\section{Aprender em momentos de adversidade}

As experiências, as aprendizagens desta pandemia devem ficar inscritas na nossa história coletiva. É dever de todos registar o vivenciado para memória futura, seja sob que forma for: escrita, imagens, filmes, objetos, desenhos, pinturas ou música.

Foi este o propósito do desafio lançado pelo Gabinete de Comunicação e Imagem, que se encontra a organizar um repositório das memórias COVID deste Centro, para que mais tarde nós e outros possamos (re)conhecer, recordar e aprender.

A pandemia por COVID-19 não terminou, nem conseguimos ainda prever o seu fim ou o seu controlo a nível mundial. Não sabemos o que nos reservam os meses ou até os anos mais próximos. No entanto, é evidente que a cultura de segurança e o conjunto de competências desenvolvidas no âmbito da segurança do paciente são essenciais à 
adaptação a um ambiente em constante mudança. A atividade das equipas responsáveis pela qualidade e segurança do paciente assenta na teoria dos sistemas, complexidade dos sistemas de saúde, teoria dos fatores humanos e metodologias de mudança e adaptação. Estas constituem ferramentas essenciais para o sucesso de um programa de mudança e no apoio a doentes, profissionais e organização (60).

\section{Conclusão}

Em segurança do paciente, a aprendizagem com os erros para evitar que voltem a acontecer é um lema. Construir estratégias e medidas que os mitiguem, que diminuam o seu impacto, caso voltem a repetir-se é um pilar essencial.

O desafio futuro é olhar para esta pandemia como uma oportunidade de aprendizagem pessoal e profissional.

Ninguém fica indiferente depois de ter experienciado uma situação com esta dimensão planetária, sejam quais forem as circunstâncias em que a tenha vivido.

É tempo de refletir e voltar aos princípios básicos como a higiene das mãos, a etiqueta respiratória e o controlo ambiental. Reforçar a necessidade de não descurar medidas de segurança, de ter redundâncias, de tornar sistemática a dupla verificação na identificação dos pacientes, na preparação e administração de medicamentos, entre muitas outras.

Em cuidados de saúde, mais especificamente em gestão do risco e segurança do paciente, nunca como agora aprender com os erros e com a adversidade fez tanto sentido e é tão necessária.

\section{Referências}

1. Direção-Geral da Saúde. COVID-19 [Internet]. [citado em 14 maio 2020]. Disponível em: https://covid19.min-saude.pt/

2. Portugal. Presidência do Conselho de Ministros, Decreto-Lei n. ${ }^{\circ} 10-A / 2020$ de 13 de março de 2020. Estabelece medidas excecionais e temporárias relativas à situação epidemiológica do novo Coronavírus - COVID 1. [citado em 3 jun. 2020]. Disponível em: https://dre.pt/application/conteudo/130243053

3. Agência para o Desenvolvimento e Coesão, I.P. [homepage na internet]. COVID-19: 10 medidas da União Europeia para lutar contra a pandemia. 7 abr. 2020 [citado em 14 maio 2020]. Disponível em: https://www.adcoesao.pt/content/covid-19-10-medidas-da-uniaoeuropeia-para-lutar-contra-pandemia 


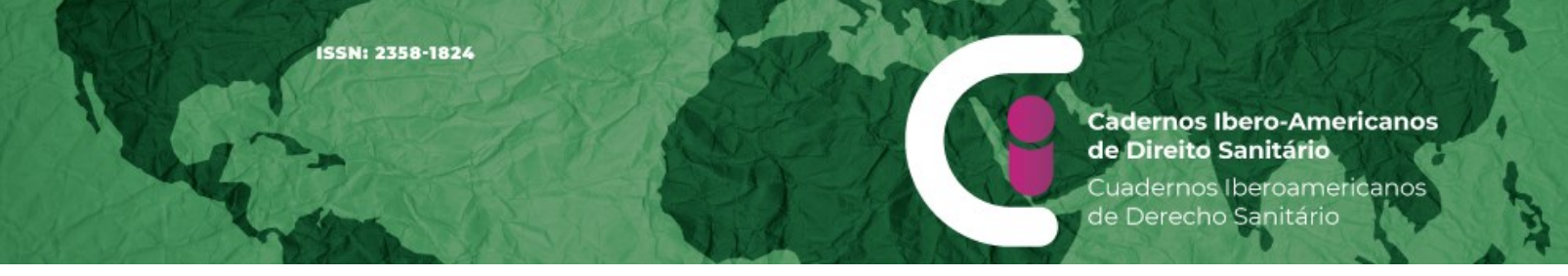

35. Wakam GK, Montgomery JR, Biesterveld BE, Brown GS. Not Dying Alone - Modern Compassionate Care in the Covid-19 Pandemic. N Engl J Med. 2020 [citado em 14 maio 2020];382(24):e88. doi https://doi.org/10.1056/NEJMp2007781

36. Rios I, Ruffini V, Montal A, Harima L, Crispim D, Arai L, et al. Virtual visits to inpatients by their loved ones during COVID-19. Clinics. 2020 [citado em 3 nov. 2020];75:eE2171. doi https://doi.org/10.6061/clinics/2020/e2171

37. Chan A, Nickson C, Rudolph J, Lee A, Joynt G. Social media for rapid knowledge dissemination: early experience from the COVID-19 pandemic. 2020 [citado em 14 maio 2020]. doi https://doi.org/10.1111/anae.15057.

38. Diniz A. O testemunho de uma enfermeira: Existe distância em tempos de pandemia? Diário de Notícias. 30 abr. 2020 [citado em 14 maio 2020]. Disponível em:

https://www.dn.pt/vida-e-futuro/o-testemunho-de-uma-enfermeira-existe-distancia-emtempos-de-pandemia-12137973.html

39. Spalluto L, Planz V, Stokes LA, Pierce R, Aronoff D, McPheeters M, et al. Transparency and trust during the coronavirus disease 2019 (COVID-19) pandemic. Journal of the American College of Radiology. 2020 [citado em 14 maio 2020]. doi https://doi.org/10.1016/j.jacr.2020.04.026

40. Rangachari $P$, Woods J. Preserving Organizational Resilience, Patient Safety, and Staff Retention during COVID-19 Requires a Holistic Consideration of the Psychological Safety of Healthcare Workers. Int. J. Environ. Res. Public Health. 2020 [citado em 3 nov. 2020]; (17):4267. doi https://doi.org/10.3390/ijerph17124267

41. Back A, Tulsky J, Arnold R. Communication skills in the age of COVID-19. 2020 [citado em 3 nov. 2020]. doi https://doi.org/10.7326/M20-1376

42. Newton PN, Bond KC. COVID-19 and risks to the supply and quality of tests, drugs, and vaccines [published correction appears in Lancet Glob Health. Lancet Global Health. 2020 [citado em 3.nov.2020];8(7):e754-e755. doi https://doi.org/10.1016/S2214-109X(20)301364

43. Sevastru S, Curtis S, Emanuel Kole L, Nadarajah P. Failure modes and effect analysis to develop transfer protocols in the management of COVID-19 patients [published online ahead of print, 2020 Apr 27]. British Journal of Anaesthesia. 2020 [citado em 14 maio 2020];S0007-0912(20)30270-1. doi https://doi.org/10.1016/j.bja.2020.04.055

44. Pryor R, Atkinson C, Cooper K, Doll M, Godbout E, Stevens MP, Bearman G. The electronic medical record and COVID-19: Is it up to the challenge? American Journal of Infection Control. 2020 [citado em 3 nov. 2020]:1-2. doi

https://doi.org/10.1016/j.ajic.2020.05.002

45. Ranney ML, Griffeth V, Jha AK. Critical Supply Shortages - The Need for Ventilators and Personal Protective Equipment during the Covid-19 Pandemic. New England Journal of Medicine. 2020 [citado em 3 nov. 2020];382(18):e41. doi

https://doi.org/10.1056/NEJMp2006141 
46. World Health Organization. Rational use of personal protective equipment for coronavirus disease (COVID-19) and considerations during severe shortages: interim guidance, 6 April 2020. World Health Organization. 2020 [citado em 22 maio 2020]. Disponível em: https://apps.who.int/iris/handle/10665/331695

47. Ma R, Deng Z, Wu M. Effects of health information dissemination on user follows and likes during COVID-19 outbreak in China: Data and content analysis. International journal of environmental research and public health. 2020 [citado em 3 nov. 2020];17(14):5081. doi https://doi.org/10.3390/ijerph17145081

48. Cartaz informativo relativo a práticas seguras na identificação do doente no contexto da pandemia por COVID-19. Centro Hospitalar Universitário Lisboa Central. 2020 [citado em 14 maio 2020]. Disponível em:

http://jintrasp/Covid19/Documents/Identificação\%20COVID\%2019-GSD.pdf

49. Ferramenta de sistematização de informação na transição de cuidados, no contexto da pandemia por COVID 19. Centro Hospitalar Universitário Lisboa Central. 2020 [citado em 14 maio 2020]. Disponível em: http://jintrasp/Covid19/Documents/Posteres\%20SegurançaISBAR_covid19\%20(3).pdf

50. Associação Portuguesa para o Desenvolvimento Hospitalar. Segurança do doente em tempos de COVID-19 [Webinar]. Lisboa; 2020 [citado em 22 maio 2020]. Disponível em: https://www.apdh.pt/evento/20

51. Centro Hospitalar Lisboa Central. Linha de atendimento, informação / orientação técnica COVID-19 [Vídeo]. Lisboa; 2020 [citado em 22 maio 2020]. Disponível em: https://www.youtube.com/watch?v=TgcUBi_j_Ds\&feature=youtu.be

52. Gavin B, Hayden J, Adamis D, McNicholas F. Caring for the Psychological Well-Being of Healthcare Professionals in the Covid-19 Pandemic Crisis. Irish Medical Journal. 2020 [citado em 21 jan. 2021];113(4):51-53. Disponível em: https://www.irishpsychiatry.ie/wpcontent/uploads/2020/04/Caring-for-the-Psychological-Well-Being-of-HealthcareProfessionals-in-the-Covid-19-Pandemic-Crisis-IMJ-2020.pdf

53. Wu AW, Connors C, Everly JR, George S. COVID-19: peer support and crisis communication strategies to promote institutional resilience. Annals of Internal Medicine. 2020 [citado em 3 nov. 2020];172(12):822-823. doi https://doi.org/10.7326/M20-1236

54. Dewey C, Hingle S, Goelz E, Linzer M. Supporting Clinicians During the COVID-19 Pandemic. Ann Intern Med. 2020 [citado em 3 nov. 2020];172:752-753. doi https://doi.org/10.7326/M20-1033

55. Cordeiro A. Os profissionais do Curry Cabral na linha da frente: "Ninguém está preparado para isto". Público. 17 maio 2020 [citado em 18 maio 2020]. Disponível em: https://www.publico.pt/multimedia/sociedade/linha-frente-curry-cabral 
56. Life Lines Team comprising. "Restricted family visiting in intensive care during COVID19." Intensive \& critical care nursing. 2020 [citado em 3 nov. 2020];102896. doi https://doi.org/10.1016/j.iccn.2020.102896

57. Bembich S, Tripani A, Mastromarino S, Di Risio G, Castelpietra E, Risso, FM. Parents experiencing NICU visit restrictions due to COVID-19 pandemic. Acta Paediatrica. 2020 [citado em 3 nov. 2020]. doi https://doi.org/10.1111/apa.15620

58. Verbeek H, Gerritsen D, Backhaus R, Boer B, Koopmans R, Hamers J. Allowing Visitors Back in the Nursing Home During the COVID-19 Crisis: A Dutch National Study Into First Experiences and Impact on Well-Being, Journal of the American Medical Directors Association. 2020 [citado em 3 nov. 2020];21(7):900-904. doi https://doi.org/10.1016/j.jamda.2020.06.020

59. Inácio AM. Das visitas virtuais aos doentes ao apoio aos profissionais. COVID-19 está a mudar os hospitais. Diário de Notícias. 12 maio 2020 [citado em 14 maio 2020].

Disponível em: https://www.dn.pt/edicao-do-dia/12-mai-2020/das-visitas-virtuais-aosdoentes-ao-apoio-aos-profissionais-covid-19-esta-a-mudar-os-hospitais-12164475.html

60. Staines A, Amalberti R, Berwick DM, Braithwaite J, Lachman P, Vincent CA. COVID-19: patient safety and quality improvement skills to deploy during the surge. Int J Qual Health Care. 2020 [citado em 3 nov. 2020];mzaa05. doi https://doi.org/10.1093/intqhc/mzaa050

\section{Colaboradores}

Ferreira CIGM e Diniz ACAM contribuíram para a concepção, redação e aprovação da versão final do artigo. Bordalo IMSVL e Leitão MJLS contribuíram para a concepção, revisão crítica e aprovação da versão final do artigo. Ramos SMSV contribuiu para a revisão crítica e aprovação da versão final do artigo.

Como citar este artigo

Ferreira CIGM, Diniz ACAM, Bordalo IMSVL, Leitão MJLS, Ramos SMSV. Podemos falar de segurança do paciente durante uma pandemia? Uma experiência portuguesa. Cadernos Ibero-Americanos de Direito Sanitário. 2021 jan./mar.;10(1):129-148. 